\title{
Note
}

\section{Axisymmetric compressible boundary layer on a long thin moving cylinder}

\author{
T.-Y. Na, Dearborn, Michigan, and I. Pop, Cluj, Romania \\ (Received August 24, 1998, revised October 26, 1998)
}

\begin{abstract}
Summary. The steady laminar boundary layer flow on a thin and isothermal moving cylinder in a viscous compressible fluid at rest is studied in this paper. The fluid considered is a model fluid in which $\varrho \sim T^{-1}$ and $\mu \sim T^{\omega}$. The transformed non-similar boundary layer equations are solved numerically using a very efficient numerical method for some values of $\omega$ and wall enthalpy $g_{w}$ parameters. The skin friction coefficient and Nusselt number are calculated for $\operatorname{Pr}=0.7$ and some values of $\omega$ and $g_{w}$.
\end{abstract}

\section{Introduction}

The study of compressible boundary layer flow over a moving wall is of interest in many branches of engineering such as, for example, in missile aerodynamics, shock generated boundary layers, aircraft response to atmospheric gusts, etc. The attenuation of a travelling shock has become important due to safety and noise considerations in high-speed ground transportation systems.

Boundary layer flows over a moving surface are of great importance in view of their relevance to a wide variety of technical applications, particularly in the manufacture of fibres in glass and polymer industries. The investigation of drying and heat transfer in such situations belongs to a separate class of problems in boundary layer theory distinguishing itself from the study of flows over static surfaces.

Sakiadis [1] was probably the first who has studied the boundary layer flow on a moving cylindrical surface in a viscous incompressible fluid at rest. Subsequently, several investigators [2]-[11] have worked on this problem under different conditions. However, very little work [12], [13] has been done on the boundary layer flow over a surface moving in a viscous compressible fluid at rest.

In this paper an attempt is made to investigate the effect of heat transfer on the compressible boundary layer in axial flow on a long thin circular cylinder which moves parallel to its axis in a fluid at rest. Owing to extreme complexity of the full compressible boundary layer equations a mathematical model is adopted which can be justified to a certain extent on physical grounds. In short, the assumption is made that the effects of compressibility are confined to the boundary layer and the main stream remains incompressible. This could be realized in practice by releasing a stream of small Mach number past a very hot body. The fluid considered is a model fluid in which $\varrho \sim T^{-1}$ and $\mu \sim T^{\omega}$, where $\varrho, T, \mu$ and $\omega$ are, respectively, 
viscosity and index in the power-law variation of viscosity. Under these assumptions, the governing differential equations are transformed into a form amenable to numerical solution. These equations are non-similar and they are solved using a very efficient finite-difference method known as Keller-box method developed by Keller and Cebeci [14]. Solutions of the velocity and enthalpy distributions are obtained from which estimates of the skin friction coefficient and local Nusselt number can be obtained.

\section{Basic equations}

We consider the steady laminar compressible boundary layer formed over a thin cylinder of infinite length and radius $a$ which moves with a constant velocity $U$ in a fluid at rest as shown in Fig. 1. We assume that the properties of the fluid vary as $\varrho \sim T^{-1}$ and $\mu \sim T^{w}$. It is also assumed that the external flow is homentropic, that the surface of the cylinder is maintained at a constant temperature $T_{w}$ and that the external flow has the uniform temperature $T_{e}$. Under these assumptions the governing equations can be written as

$\frac{\partial(\varrho r u)}{\partial x}+\frac{\partial(\varrho r v)}{\partial y}=0$

$\varrho\left(u \frac{\partial u}{\partial x}+v \frac{\partial u}{\partial y}\right)=\frac{1}{r} \frac{\partial}{\partial y}\left(\mu r \frac{\partial u}{\partial y}\right)$

$\varrho C_{p}\left(u \frac{\partial T}{\partial x}+v \frac{\partial T}{\partial y}\right)=\frac{1}{r} \frac{\partial}{\partial y}\left(k r \frac{\partial T}{\partial y}\right)$

subject to the boundary conditions

$$
\begin{aligned}
& u(x, 0)=U, \quad v(x, 0)=0, \quad T(x, 0)=T_{w}, \\
& u(x, \infty)=0, \quad T(x, \infty)=T_{e}, \\
& u(0, y)=0, \quad v(0, y)=0, \quad T(0, y)=T_{e} .
\end{aligned}
$$

Here $x$ measures the distance along the axis of the cylinder from the extrusion slot, $y$ measures the normal distance from the surface of the cylinder, and $r=a+y$ is the normal distance from the axis of the cylinder, $u$ and $v$ are the velocity components in the axial and normal direction, respectively, $T$ is the fluid temperature, and $k$ is the thermal conductivity of the fluid.

Further, introducing the new variables (see Choi [12])

$$
\bar{x}=x, \quad \bar{y}=y+\frac{y^{2}}{2 a}, \quad \bar{u}=u, \quad \bar{v}=\left(\frac{r}{a}\right) v
$$

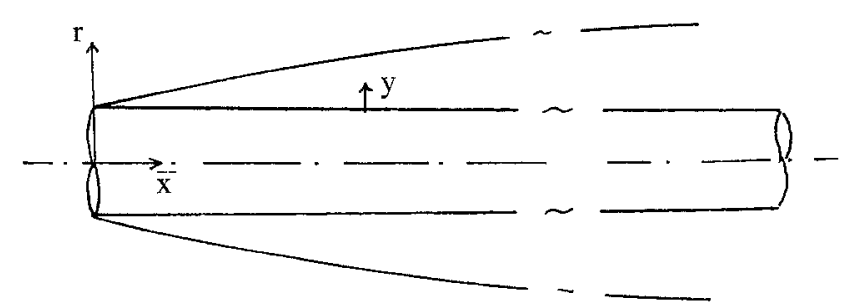

Fig. 1. Physical model and coordinate system 
the equation of continuity is satisfied if the stream function $\psi$ is defined as

$\bar{u}=\frac{1}{a}\left(\frac{\varrho_{e}}{\varrho}\right) \frac{\partial \psi}{\partial \bar{y}}, \quad \bar{v}=-\frac{1}{a}\left(\frac{\underline{\varrho}_{e}}{\varrho}\right) \frac{\partial \psi}{\partial \bar{x}}$.

Using (5), Eqs. (2) and (3) can be cast into a quasi-two-dimensional form,

$$
\begin{aligned}
& \varrho\left(\bar{u} \frac{\partial \bar{u}}{\partial \bar{x}}+\bar{v} \frac{\partial \bar{u}}{\partial \bar{y}}\right)=\frac{\partial}{\partial \bar{y}}\left[\left(1+\frac{2}{a} \bar{y}\right) \mu \frac{\partial \bar{u}}{\partial \bar{y}}\right], \\
& \varrho C_{p}\left(\bar{u} \frac{\partial T}{\partial \bar{x}}+\bar{v} \frac{\partial T}{\partial \bar{y}}\right)=\frac{\partial}{\partial \bar{y}}\left[\left(1+\frac{2}{a} \bar{y}\right) k \frac{\partial T}{\partial \bar{y}}\right],
\end{aligned}
$$

subject to the same boundary conditions (4).

Equations (7) and (8) are further transformed into a nondimensional form by introducing the variables

$$
\begin{aligned}
& \xi=\left(\frac{2 \nu_{e} \bar{x}}{U a^{2}}\right)^{2}, \quad Y=\frac{\bar{y}}{a}, \quad \eta=\frac{1}{\xi} \int_{0}^{Y} \frac{\varrho}{\varrho_{e}} d Y, \\
& \psi=\operatorname{Ua\xi } f(\xi, \eta), \quad h=C_{p} T, \quad g=\frac{h}{h_{e}}, \quad N=\frac{\varrho \mu}{\varrho_{e} \mu_{e}} .
\end{aligned}
$$

Thus, we have after some manipulations

$$
\begin{aligned}
& \frac{\partial}{\partial \eta}\left[(1+2 Y) g^{\omega-1} \frac{\partial^{2} f}{\partial \eta^{2}}\right]+f \frac{\partial^{2} f}{\partial \eta^{2}}=\xi\left\{\frac{\partial f}{\partial \eta} \frac{\partial^{2} f}{\partial \xi \partial \eta}-\frac{\partial f}{\partial \xi} \frac{\partial^{2} f}{\partial \eta^{2}}\right) \\
& \frac{1}{\operatorname{Pr}} \frac{\partial}{\partial \eta}\left[(1+2 Y) g^{\omega-1} \frac{\partial g}{\partial \eta}\right]+f \frac{\partial g}{\partial \eta}=\xi\left\{\frac{\partial f}{\partial \eta} \frac{\partial g}{\partial \xi}-\frac{\partial f}{\partial \xi} \frac{\partial g}{\partial \eta}\right) \\
& Y=\xi \int_{0}^{\eta} g d \eta
\end{aligned}
$$

subject to the boundary conditions

$f=0, \quad \frac{\partial f}{\partial \eta}=1, \quad g=g_{w} \quad$ at $\quad \eta=0 ; \quad \frac{\partial f}{\partial \eta}=0, \quad g=1 \quad$ as $\quad \eta \rightarrow \infty$,

where

$\frac{\varrho_{e}}{\varrho}=\frac{h}{h_{e}}=g, \quad \frac{\mu}{\mu_{e}}=\left(\frac{h}{h_{e}}\right)^{\omega}, \quad N=g^{\omega-1}$.

Here $h$ is the specific enthalpy and $\operatorname{Pr}=\mu C_{p} / k$ is the Prandtl number which is taken as a constant across the boundary layer because its variation in the boundary layer for most atmospheric flight problems is less than 5 per cent (see Wortman et al. [13]).

Equations (10) and (11) can be reduced to two limiting cases: (i) constant property formulation is obtained if $g=1$ (i.e., $\varrho \mu=$ constant). In this case $Y=\xi \eta$ and Eq. (10) reduces to that of Karnis and Pechoc [5]; (ii) if $Y=0$, Eqs. (10) and (11) reduce to the following ordinary differential equations:

$$
\begin{aligned}
& \left(g^{\omega-1} f^{\prime \prime}\right)+f f^{\prime \prime}=0, \\
& \left(g^{\omega-1} g^{\prime}\right)+\operatorname{Pr} f g^{\prime}=0
\end{aligned}
$$

along with the boundary conditions

$f(0)=0, \quad f^{\prime}(0)=1, \quad g(0)=g_{w} ; \quad f^{\prime}(\infty)=0, \quad g(\infty)=1$. 
This case can be realized physically of $\delta / a \ll 1$ which is equivalent to the condition $\xi \rightarrow 0$, where $\delta$ is the boundary layer thickness. Thus, by taking $\xi$ sufficiently small the solution for the axisymmetric compressible boundary layer flow along a thin moving cylinder can be matched to its two-dimensional counterpart. This limiting case is characterized by the compressible boundary layer flow on a moving semi-infinite flat plate.

The skin friction coefficient and the local Nusselt number are defined as

$C_{f}=\frac{-\tau_{w}}{\varrho_{e} U^{2}}, \quad \mathrm{Nu}=\frac{C_{p} \bar{x} q_{w}}{k_{e}\left(h_{e}-h_{w}\right)}$,

where $\tau_{w}$ and $q_{w}$ are the skin friction and heat transfer from the surface of the cylinder, and they are given by

$\tau_{w}=\mu_{w}\left(\frac{\partial \bar{u}}{\partial \bar{y}}\right)_{\bar{y}=0}, \quad q_{w}=\frac{\mu_{w}}{\operatorname{Pr}}\left(\frac{\partial h}{\partial \bar{y}}\right)_{\bar{y}=0}$.

Using the variables (9), we obtain

$C_{f} \operatorname{Re}_{x}^{1 / 2}=g_{w}^{\omega-1}\left(-\frac{\partial^{2} f}{\partial \eta^{2}}\right)_{\eta=0}$,

$\frac{\mathrm{Nu}^{\mathrm{u}}}{\operatorname{Re}_{x}^{1 / 2}}=\frac{g_{w}^{\omega-1}}{1-g_{w}}\left(\frac{\partial g}{\partial \eta}\right)_{\eta=0}$,

where $\operatorname{Re}_{x}=U \bar{x} / \nu_{e}$ is the local Reynolds number.

\section{Results and discussion}

Equations (10) and (11) along with the boundary conditions (13) have been solved numerically using the Keller-box scheme [14]. To test the accuracy of the present method, we have compared the initial slope of $f^{\prime \prime}(0)$ for $\omega=1$ from this work with the value from $\mathrm{Na}$ and Pop [15]. The present method gives $f^{\prime \prime}(0)=-0.627557$, while that of Na and Pop [15] is $f^{\prime \prime}(0)=-0.627559$. We are therefore confident that the present results are very well done.

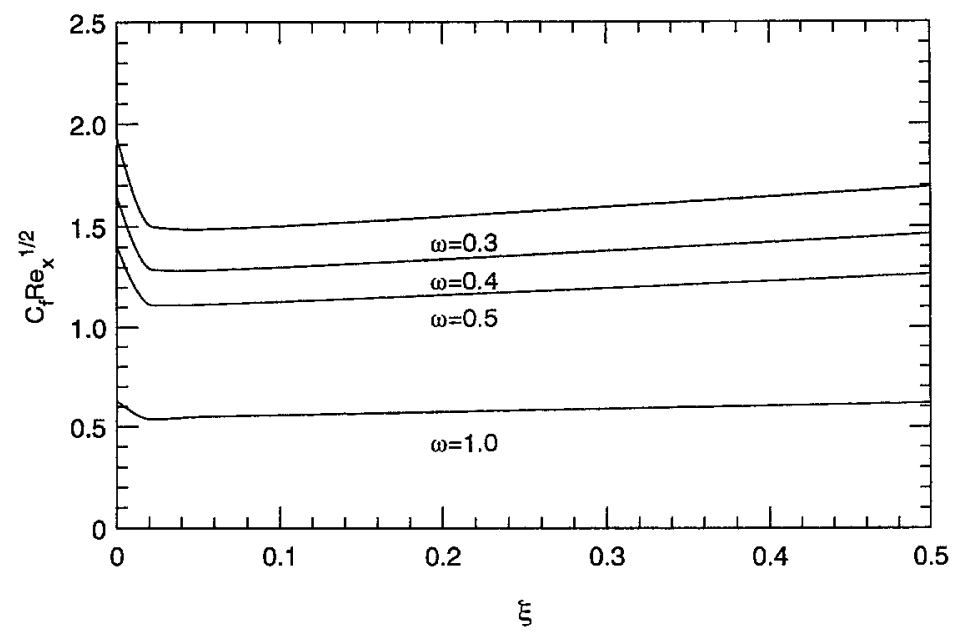

Fig. 2. Local skin friction coefficient for $\operatorname{Pr}=0,7, g_{w}=0.2$ 
Axisymmetric compressible boundary layer

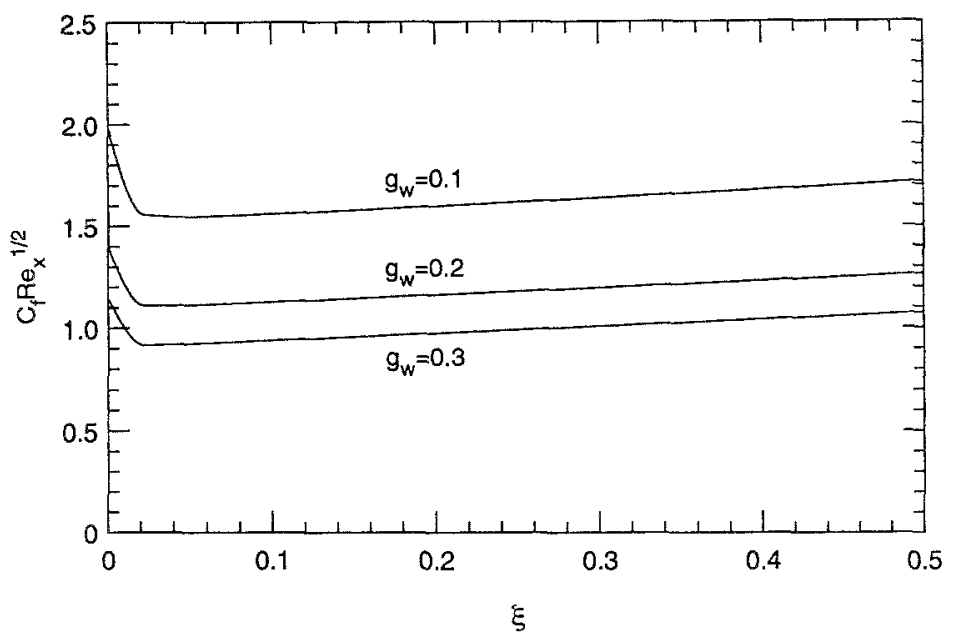

Fig. 3. Local skin friction coefficient for $\operatorname{Pr}=0.7, \omega=0.5$

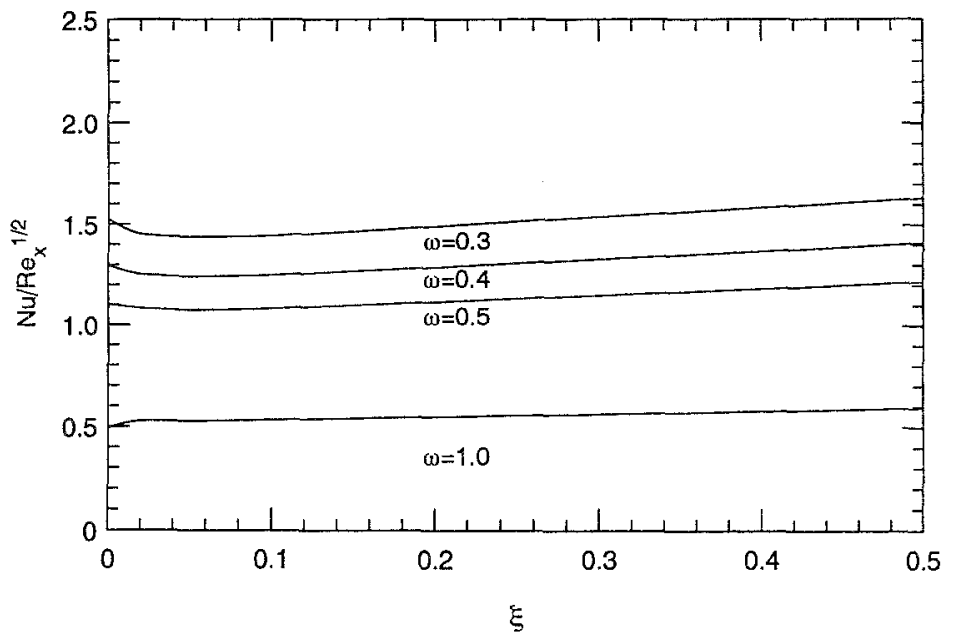

Fig. 4. Local Nusselt number for $\operatorname{Pr}=0.7, g_{w}=0.2$

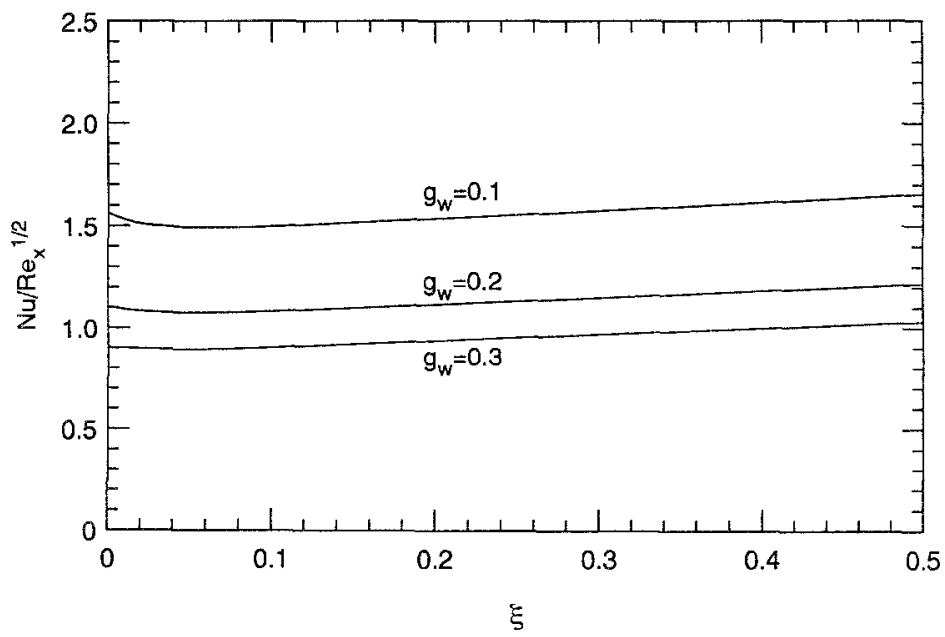

Fig. 5. Local Nusselt number for $\operatorname{Pr}=0.7, \omega=0.5$ 
The variation of $C_{f} \operatorname{Re}_{x}^{1 / 2}$ and $\mathrm{Nu}_{x} / \mathrm{Re}_{x}^{1 / 2}$ is shown in Figs. $2-5$ versus $\xi$ for $\operatorname{Pr}=0.7$ and different values of $\omega$ and $g_{w}$. The results for an incompressible fluid $(\omega=1)$ were also included in Figs. 2 and 4 for the sake of comparison.

We notice from these figures that the variable fluid properties affect significantly the skin friction and heat transfer rate. Thus, both $C_{f}$ and Nu decrease with the increase of the parameters $\omega$ and $g_{w}$. The deviation of variable property solutions against the constant property case is believed to be the result of fluid property interactions rather than the result of the individual role played by each property. It is found, however, that a solution does not exist for the thermal boundary layer equation (11) subject to the boundary conditions (13) when the wall enthalpy $g_{w}=1$ and $\omega \neq 1$.

It is also seen from these figures that the axial nondimensional parameter $\xi$ affects the skin friction coefficient and Nusselt number more significantly only near the slot. Far downstream $C_{f}$ and Nu remain almost constant with $\xi$.

\section{References}

[1] Sakiadis, B. C.: Boundary layer behavior on continuous surfaces: III. The boundary layer on a continuous cylindrical surface. A.I.Ch.E. J. 467-470 (1961).

[2] Rotte, J. M., Beek, W. J.: Some models for the calculation of heat transfer coefficients to a continuous cylinder. Chem. Eng. Sci. 24, 705-716 (1969).

[3] Bourne, D. E., Elliston, D. G.: Heat transfer through the axially symmetric boundary layer on a moving circular fibre. Int. J. Heat Mass Transfer 13, 583-593 (1970).

[4] Gampert, B.: Berechnung des Wärmeübertrages an einem in ruhendem Fluid kontinuierlich bewegten schlanken Kreiszylinder für kleine Werte des Krümmungsparameters auf der Basis von Reihenansätzen. ZAMM 54, T 118 (1974).

[5] Karnis, J., Pechoc, V.: The thermal laminar boundary layer on a continuous cylinder. Int. J. Heat Mass Transfer 21, 43-47 (1978).

[6] Lin, H. T., Shih, Y. P.: Laminar boundary layer heat transfer along static and moving cylinders. J. Chin. Inst. Eng. 3, $73-79(1980)$.

[7] Pop, I., Kumari, M., Nath, G.: Non-Newtonian boundary layers on a moving cylinder. Int. J. Eng. Sci. 28, 303-312 (1990).

[8] Eswara, A. T., Nath, G.: Unsteady forced convection laminar boundary layer flow over a moving longitudinal cylinder. Acta Mech. 93, 13-28 (1992).

[9] Pop, I., Watanabe, T., Taniguchi, H.: Laminar boundary layer flow and heat transfer along a moving cylinder with suction or injection. Techn. Mech. 15, 99- 106 (1995).

[10] Na, T.-Y., Pop, I.: Flow and heat transfer over a longitudinal circular cylinder moving in parallel or reversely to a free stream. Acta Mech. 118, 185-195 (1996).

[11] Wang, T.-Y.: Convective heat transfer between a moving cylinder and flowing non-Newtonian fluids. Int. Comm. Heat Mass Transfer 23, 101-114 (1996).

[12] Choi, I. G.: The effect of variable properties of air on the boundary layer for a moving continuous cylinder. Int. J. Heat Mass Transfer 25, 597-602 (1982).

[13] Kumari, M., Takhar, H. S., Nath, G.: Compressible boundary layer flow over a moving surface with a magnetic field. Rev. Roum. Sci. Techn. Mec. Appl. 40, 25-32 (1995).

[14] Keller, H. B., Cebeci, T.: Accurate numerical methods for boundary layers. Part I. Two-dimensional laminar flows. In: Proc. 2nd Int. Conf. Numerical Methods in Fluid Dynamics. Lecture Notes in Physics, Vol. 8. New York: Springer 1971.

[15] Na, T.-Y., Pop, I.: A note to the solution of Cheng-Minkowycz equation arising in free convection flow in porous media. Int. Comm. Heat Mass Transfer 23, 697-703 (1996).

Authors' addresses: Prof. T.-Y. Na, Department of Mechanical Engineering, University of Michigan Dearborn, Dearborn, MI 48128-1491, USA, and Prof. I. Pop, Faculty of Mathematics, University of Cluj, R-3400 Cluj, CP 253, Romania 